\title{
Razón de Compresión y Aproximaciones Lineales en el Ciclo de Curzon y Ahlborn
}

Delfino Ladino-Luna y Ricardo T. Páez-Hernández

Universidad Autónoma Metropolitana-Atzcapotzalco, Área de Física de Procesos Irreversibles, Dpto. de Ciencias Básicas, Av. San Pablo 180, Col. Reynosa, 02200, Atzcapotzalco, D. F.-México (e-mail: dll@correo.azc.uam.mx; phrt@correo.azc.uam.mx)

Recibido Oct. 26, 2009; Aceptado Ene. 08, 2010; Versión Final recibida Feb. 12, 2010

\section{Resumen}

Para el llamado ciclo endorreversible de Curzon y Ahlborn se encuentran aproximaciones lineales para su eficiencia en términos de la razón de los volúmenes máximo y mínimo alcanzados durante el ciclo (la razón de compresión), considerando el tiempo de duración de los procesos adiabáticos. Se analiza el comportamiento de la razón de compresión respecto de la razón de temperaturas de las fuentes fría y caliente. Se encuentra que existe una relación entre la razón de compresión y la razón de temperaturas de las fuentes fría y caliente, que mejora la eficiencia del modelo utilizado. También se encuentra que una aproximación a segundo orden debe llevar a un mayor acercamiento entre la eficiencia aproximada y los valores reportados en la literatura.

Palabras clave: eficiencia, endorreversibilidad, razón de compresión, ciclos Curzon and Ahlborn

\section{Compression Ratio and Linear Approximations in the Curzon and Ahlborn Cycle}

\begin{abstract}
Linear approximations of the efficiency as function of maximum and minimum volumes ratio (compression ratio) in the endorreversible Curzon and Ahlborn cycle are found, taking into account the time of duration of the adiabatic processes in this cycle. The performance of the compression ratio versus the temperatures of the cold and hot reservoirs ratio is analyzed. It is found that there exist a relation between compression ratio and temperatures of the cold and hot reservoirs ratio that improve the efficiency of the used model. It is also shown that a second order approximation should give a closer relation between the approximate efficiency and literature values.
\end{abstract}

Keywords: efficiency, endoreversibility, compression ratio, Curzon and Ahlborn cycle 


\section{INTRODUCCIÓN}

En el contexto de la termodinámica clásica de equilibrio el ciclo más eficiente posible es el conocido ciclo de Carnot. Una generalización de este ciclo, como modelo de una máquina térmica, considerando la diferencia entre las temperaturas de los almacenes y las de la substancia de trabajo, fue introducida por Curzon y Ahlborn (1975), figura 1, conocido ahora como ciclo de Curzon y Ahlborn. A partir de esta consideración, para las temperaturas de los almacenes frío y caliente, respectivamente, $T_{1}$ y $T_{2}$, con $T_{1}>T_{2}$, estos autores encontraron para este ciclo la eficiencia:

$\eta_{C A}=1-\sqrt{T_{2} / T_{1}}$,

A partir de la publicación del trabajo de Curzon y Ahlborn se publicaron nuevos resultados en la ahora llamada Termodinámica de Tiempos Finitos (Salamon et al, 1977 y 1980; Gutkowicz-Krusin et al, 1978; Rubin, 1979, 1980; etc), recuperando la expresión (1) por diversos métodos, maximizando diferentes funciones objetivo, a saber, potencia, eficiencia ó producción de entropía. Particularmente Salamon et al (1980) introdujeron la producción de entropía en la optimización del ciclo. Posteriormente Ondrechen et al (1983), Torres (1988) y Angulo-Brown (1991a) utilizaron la minimización de la entropía producida, durante los intercambios de calor entre el sistema y sus alrededores para recuperar el resultado de Curzon y Ahlborn (1975). Más aún, Angulo-Brown (1991b) propuso una función que combina la potencia de salida del ciclo reversible con la producción de entropía del ciclo endorrevresible, la función ecológica,

$E=P-T_{2} \sigma$

La maximización de $E$ lleva a la llamada eficiencia ecológica, $\eta_{E}$, en función de las mismas temperaturas de la eficiencia de Curzon y Ahlborn, ecuación (1). Definiendo $\varepsilon \equiv T_{2} / T_{1}$, se tiene

$\eta_{E}=1-\sqrt{\frac{1}{2}\left(\varepsilon+\varepsilon^{2}\right)}$.

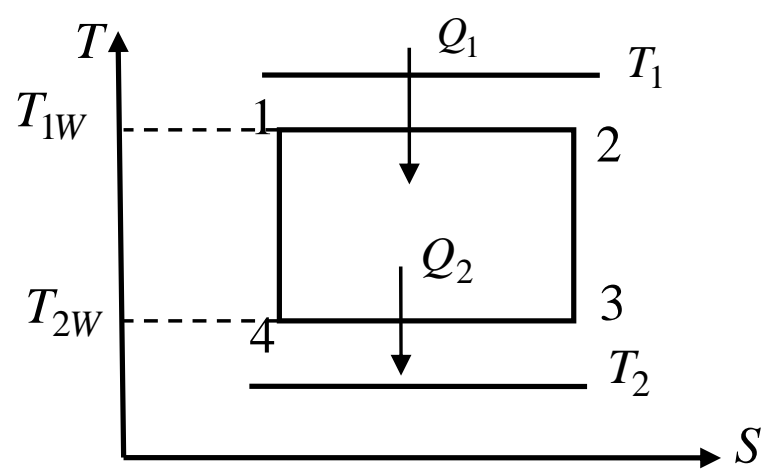

Fig. 1. Ciclo endorreversible en el plano entropía $S$ vs temperatura $T$ (Ciclo de Curzon y Ahlborn).

En los trabajos antes citados, y otros semejantes, excepto en Gutkowicz-Krusin et al (1978), se analiza el ciclo de Curzon y Ahlborn en el caso donde el tiempo de duración de los procesos adiabáticos es irrelevante. Pero, en los sistemas reales dicho tiempo no es cero. Así, GutkowiczKrusin et al (1979) y Agrawal et al (1994) propusieron un tiempo de duración de los procesos adiabáticos en función del tiempo de los procesos isotérmicos.

Particularmente Gutkowicz-Krusin et al (1979) lograron hallar una expresión para la eficiencia, maximizando la potencia de salida de la máquina, en función de la razón de compresión $r_{C}=V_{3} / V_{1}$, donde $V_{3}$ y $V_{1}$ son los volúmenes máximo y mínimo subtendidos por la máquina. Hallaron además que la eficiencia (1) es una cota superior, que corresponde a una razón de compresión infinita. 
Agrawal y Menon $(1990,1993)$ utilizaron este procedimiento para analizar un refrigerador de Carnot a máxima potencia de enfriamiento. Más recientemente, Ladino-Luna y de la Selva (2000) mostraron a la eficiencia ecológica como una cota superior para la eficiencia del ciclo de Curzon y Ahlborn, cuando la función objetivo es la función ecológica. En todos estos trabajos se supone una ley de la transferencia de calor como la ley de enfriamiento de Newton.

Pero, así como la forma de la eficiencia cambia con la función objetivo utilizada, como se colige de las ecuaciones (1) y (2), una ley de transferencia de calor diferente tiene también un efecto en la forma de la eficiencia. Así, Yan y Chen (1990), Angulo-Brown y Páez-Hernández (1993), ÁriasHernández y Angulo-Brown (1994) y Chen et al (1999), entre otros, estudiaron el efecto de considerar una ley de transferencia de calor diferente a la ley de enfriamiento de Newton. Recientemente Ladino-Luna (2008) utilizó una ley de transferencia tipo Dulong y Petit para hallar resultados más generales que los hallados en Gutkowicz-Krusin et al (1979) y Ladino-Luna y de la Selva (2000).

En el presente trabajo se toma en cuenta la duración de los procesos adiabáticos en el ciclo de Curzon y Ahlborn, maximizando la potencia y la función ecológica, para obtener una aproximación a primer orden para la eficiencia en función de la razón de compresión. Se encuentra la eficiencia como función de la razón de compresión y de la razón de las temperaturas de los almacenes, y se obtiene una relación de tres parámetros, $F\left(\eta, r_{C}, \varepsilon\right)=0$, con la misma estructura independientemente de obtenerse al maximizar la función potencia de salida ó la función ecológica. Se supone gas ideal como substancia de trabajo y se utilizan los resultados obtenidos por Gutkowicz-Krusin et al (1979) y Ladino-Luna y de la Selva (2000). Se asume la ley de transferencia de calor de Newton y una ley de transferencia de calor no lineal tipo Newton. Para fines ilustrativos, se usan los valores de la eficiencia de plantas de potencia reportados en Curzon y Ahlborn (1975) y Angulo-Brown (1991b).

\section{EFICIENCIA EN FUNCIÓN DE UNA RAZÓN DE TEMPERATURAS.}

El conocido ciclo de Carnot corresponde al caso en que las temperaturas de las fuentes y las del sistema, en la figura 1, cumplen $T_{1}=T_{1 W}$ y $T_{2}=T_{2 W}$, donde $T_{1 W}$ y $T_{2 W}$ son las temperaturas mayor y menor, respectivamente, de la substancia de trabajo. Además, la definición de eficiencia, de cualquier máquina térmica es,

$\eta=\frac{Q_{1}-Q_{2}}{Q_{1}}$

donde los calores absorbido y cedido por la máquina son $Q_{1}$ y $Q_{2}$, y su eficiencia cumple

$\eta \leq 1-T_{2} / T_{1}$

siendo la eficiencia del ciclo de Carnot $\eta_{C}=1-T_{2} / T_{1}$. Nótese que las expresiones (1), (3) y la eficiencia de Carnot son funciones del parámetro $\varepsilon \equiv T_{2} / T_{1}$. Así, la eficiencia se puede escribir como $\eta=1-z(\varepsilon)$. El problema es hallar una función $z(\varepsilon)$, tan complicada o simple como indique la ley de transferencia de calor usada, de manera que se obtenga la función $\eta=\eta(\varepsilon)$. Así, suponiendo la misma conductancia térmica para el calor absorbido y para el calor cedido, y que la transferencia de calor sucede como la ley de enfriamiento de Newton, se tiene, para los procesos isotérmicos,

$$
\frac{d Q_{i}}{d t}=(-1)^{i-1} \alpha\left(T_{i}-T_{1 W}\right) ; i=1,2
$$

Para los procesos adiabáticos, no instantáneos, se suponen tiempos de duración como función de los tiempos de duración de los procesos isotérmicos, obtenidos por integración de (6). La substancia de trabajo será un gas ideal. 


\section{MODELO DE GUTKOWICZ-KRUSIN, PROCACCIA Y ROSS}

Gutkowicz-Krusin et al (1978) tomaron explícitamente en cuenta el tiempo de duración de cada uno de los procesos indicados en el ciclo de Curzon y Ahlborn (figura 1). Tomando como máquina térmica un cilindro con un pistón móvil encerrando un gas ideal, y asumiendo que los tiempos para los procesos isotérmicos, calculados por simple integración de (6), se pueden escribir como

$t_{1}=f_{1} \ln \left(V_{2} / V_{1}\right) \quad$ y $t_{3}=f_{2} \ln \left(V_{3} / V_{4}\right)$,

definiendo los parámetros $f_{1} \equiv \frac{R T_{1 W}}{\alpha}\left(T_{1}-T_{1 W}\right)^{-1}$ y $f_{2} \equiv \frac{R T_{2 W}}{\alpha}\left(T_{2 W}-T_{2}\right)^{-1} . R$ es la constante general de los gases ideales y $\alpha=$ cte. es la conductancia térmica. Los tiempos de duración de los procesos adiabáticos se construyen como

$t_{2}=f_{1} \ln \left(V_{3} / V_{2}\right)$ y $t_{4}=-f_{2} \ln \left(V_{1} / V_{4}\right)$.

Suponiendo que se cumple la llamada hipótesis de endorreversibilidad, $Q_{1} / T_{1 W}=Q_{2} / T_{2 W}$, y puesto que el tiempo total de duración del ciclo está dado por $t_{\text {tot }}=t_{1}+t_{2}+t_{3}+t_{4}$, se obtiene la expresión para la potencia de salida de la máquina, definida por $P \equiv W / t_{\text {tot }}$, donde $W$ es el trabajo producido durante el ciclo y con los cambios de variables $x=T_{1 W} / T_{1}$ y $\quad z=T_{2 W} / T_{1 W}, \quad \lambda \equiv\left[(\gamma-1) \ln r_{C}\right]^{-1}$ y $\gamma=C_{P} / C_{V}$, en la forma

$P=\alpha T_{1}(1-z)(1+\lambda \ln z)\left[\frac{1}{1-x}+\frac{z}{z x-\varepsilon}\right]^{-1}$.

De las condiciones para máxima potencia, con las nuevas variables $x$ y $z$, a saber, $\frac{\partial P}{\partial x}=0$ y $\frac{\partial P}{\partial z}=0$, se tienen las expresiones,

$x=\frac{z+\varepsilon}{2 z}$,

$[\lambda(1-z)-z(1+\lambda \ln z)](z-\varepsilon)(z x-\varepsilon)-z(z-1)(1+\lambda \ln z)=0$,

respectivamente, de donde se encuentra la eficiencia a potencia máxima como la expansión

$\eta_{P}=1-\left\{\sqrt{\varepsilon}+\frac{1}{2}(1-\sqrt{\varepsilon})^{2} \lambda+\frac{1}{4}(1-\sqrt{\varepsilon})^{2}\left[\frac{(1-\sqrt{\varepsilon})^{2}}{2 \sqrt{\varepsilon}}-\ln \varepsilon\right] \lambda^{2}+O\left(\lambda^{3}\right)\right\}$.

La expresión encerrada por los corchetes es $z(\varepsilon)$. Se verifica que para $r_{C} \rightarrow \infty$ y $\lambda \rightarrow 0$ se recupera la eficiencia de Curzon y Ahlborn, dada ahora como $\eta_{C A} \equiv \eta_{P}(\lambda=0)=\eta_{P O}$. Además, a partir de (11), se tiene la aproximación lineal, donde el coeficiente del término lineal en $\lambda$ es $b_{P}=\frac{1}{2}(1-\sqrt{\varepsilon})^{2}$,

$\eta_{P L}(\lambda)=1-\sqrt{\varepsilon}-\frac{1}{2}(1-\sqrt{\varepsilon})^{2} \lambda$

\section{EFICIENCIA ECOLÓGICA.}

La función ecológica también se puede escribir en términos de las variables $x$ y $z$; y de los parámetros $\lambda$ y $\varepsilon$, de manera semejante a la potencia, dada en (9). Si $\sigma$ es la producción de entropía y $\Delta S$ el cambio de entropía durante el intercambio de calor, $\sigma \equiv \Delta S / t_{\text {tot }}$, se tiene que, 
$\sigma=\frac{1}{t_{\text {tot }}}\left(Q_{1} / T_{1}+Q_{2} / T_{22}\right)$

que, en términos de las variables $x$ y $z$, y de los parámetros $\lambda$ y $\varepsilon$, se escribe como,

$\sigma=\alpha \frac{T_{1}}{T_{2}}(1+\lambda \ln z)(z-\varepsilon)\left[\frac{1}{1-x}+\frac{z}{z x-\varepsilon}\right]^{-1}$.

Substituyendo (9) y (14) en (2), la función ecológica está dada ahora por la expresión

$E=\alpha T_{1}(1+\varepsilon-2 z)(1+\lambda \ln z)\left[\frac{1}{1-x}+\frac{z}{z x-\varepsilon}\right]^{-1}$.

Las condiciones de maximización para (15), $\frac{\partial E}{\partial x}=0 \quad$ y $\frac{\partial E}{\partial z}=0$, permiten obtener la variable $x$ como en (10a), y una expresión equivalente a la expresión (10b),

$[2(1+\lambda \ln z) z-\lambda(1+\varepsilon-2 z)](z-\varepsilon)(z x-\varepsilon)-\varepsilon z(1+\varepsilon-2 z)(1+\lambda \ln z)(1-x)=0$,

de donde se obtiene $z$ en potencias de $\lambda$ como $z=z_{E}(\varepsilon, \lambda)$. Así, se tiene la eficiencia ecológica,

$\eta_{E}(\lambda)=1-\left\{\sqrt{\frac{\varepsilon^{2}+\varepsilon}{2}}+\left(\frac{1}{4}(1+3 \varepsilon)-\sqrt{\frac{\varepsilon^{2}+\varepsilon}{2}}\right) \lambda+\left(\frac{\sqrt{2}(1+3 \varepsilon)}{32 \sqrt{\varepsilon^{2}+\varepsilon}}-\frac{1}{2} \ln \sqrt{\frac{\varepsilon^{2}+\varepsilon}{2}}\right)\left(+3 \varepsilon-4 \sqrt{\frac{\varepsilon^{2}+\varepsilon}{2}}\right) \lambda^{2}+O\left(\lambda^{3}\right)\right\}$.

Para recuperar la eficiencia ecológica (3) basta tomar los límites $r_{C} \rightarrow \infty$ y $\lambda \rightarrow 0$.

La correspondiente aproximación lineal de la eficiencia ecológica, donde $b_{E}$ es el coeficiente del término lineal en $\lambda$, queda ahora como

$\eta_{E L}(\lambda)=1-\sqrt{\frac{\varepsilon^{2}+\varepsilon}{2}}-\left[\frac{1}{4}(1+3 \varepsilon)-\sqrt{\frac{\varepsilon^{2}+\varepsilon}{2}}\right] \lambda, \mathrm{y}, b_{E}=\frac{1}{4}(1+3 \varepsilon)-\sqrt{\frac{1}{2}\left(\varepsilon^{2}+\varepsilon\right)}$

\section{RAZÓN DE COMPRESIÓN Y RAZÓN DE TEMPERATURAS.}

Como puede observarse, la aproximación lineal de la eficiencia, independientemente de que se haya maximizado la potencia ó la función ecológica, tiene la forma,

$\eta_{I L}(\varepsilon, \lambda)=\eta_{I O}-b_{I}(\varepsilon) \lambda=\eta_{I O}-b_{I}(\varepsilon) \frac{1}{(\gamma-1) \ln r_{C}}$

con $\lambda=\left[(\gamma-1) \ln r_{C}\right]^{-1}$, donde el subíndice $I$ se substituye por $P$ ó $E$, según se maximice la función potencia ó la función ecológica. Esto es, si se maximiza la potencia se tiene $\eta_{P L}, \eta_{P O}$ y $b_{P}$; y si se maximiza la función ecológica se tiene $\eta_{E L}, \eta_{E O}$ y $b_{E}$. Ahora, para un valor dado de la eficiencia se tiene $r_{C}=r_{C}(\varepsilon)$, y su forma general, a partir de (19), se obtiene como

$r_{C}=\exp \left\{\frac{b_{I}}{(\gamma-1)\left(\eta_{I O}-\eta_{I L}\right)}\right\}$. 
Tomando la eficiencia $\eta_{I O}$ como la calculada en Curzon y Ahlborn (1975) y Angulo-Brown (1991b), respectivamente, se tiene el intervalo de valores para la aproximación lineal de la eficiencia $\eta_{I L}$, a saber, $0<\eta_{I L}<\eta_{I O}$. Dado un valor de la eficiencia $\eta_{I L}$ es posible encontrar también un intervalo $0<\varepsilon<1$, para el cual $r_{C}$ cumple $r_{C}>1$, lo que permite hallar, a partir de (20) la relación,

$$
\frac{b_{I}}{(\gamma-1)\left(\eta_{I O}-\eta_{I L}\right)}>0
$$

que lleva a la desigualdad $\eta_{I L}<\eta_{I O}$, como una condición necesaria, pues $\eta_{I O}$ debe ser una cota superior para $\eta_{I L}$. Para el caso de gases monoatómicos, $\gamma=1.67$, con $\eta_{I L}$ como parámetro variable, se obtiene la variación de $r_{C}$, observándose que $r_{C} \rightarrow \infty$ cuando $\eta_{I L} \rightarrow \eta_{I O}$, como debe ser.

Para cada par de temperaturas dadas en Curzon y Ahlborn (1975) y Angulo-Brown (1991b), se tiene la variación de $r_{C}$ a partir de (20). Por ejemplo en el caso de la planta West Turrock las temperaturas de los almacenes, en grados Kelvin, son $T_{1}=838$ y $T_{2}=298$, con $\eta_{P O}=\eta_{C A}=0.403367$. La figura 2 muestra la variación de $r_{C}$ respecto de $\eta_{P L}$. Usando la función ecológica, para la misma planta, y las mismas temperaturas, $\eta_{E O}=\eta_{g i}=0.403367$, obtenida en Ladino-Luna y de la Selva (2000), la figura 3 muestra el comportamiento de la razón de compresión. Obsérvese que en ambos casos existe un valor mínimo de la razón de compresión, mayor de 1. La figura 4 muestra esta afirmación, con el segmento escogido (encerrado por las líneas discontinuas) de la figura 2.

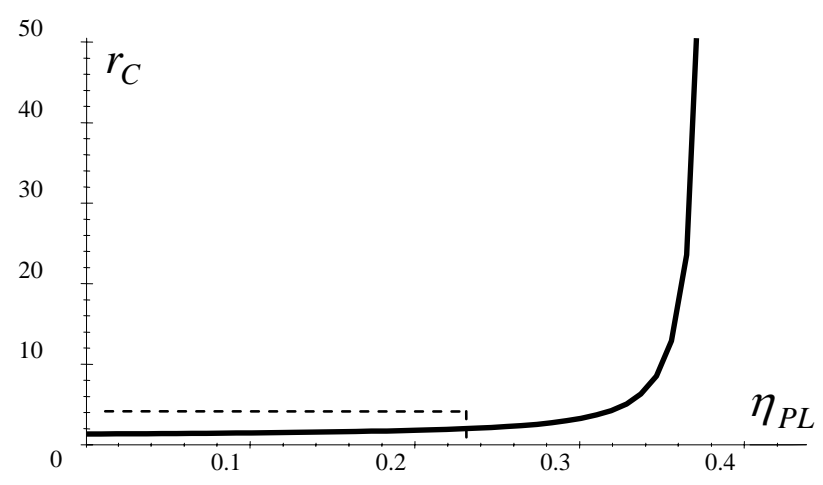

Fig. 2. Variación de $r_{C}$ respecto de $\eta_{P L}$.

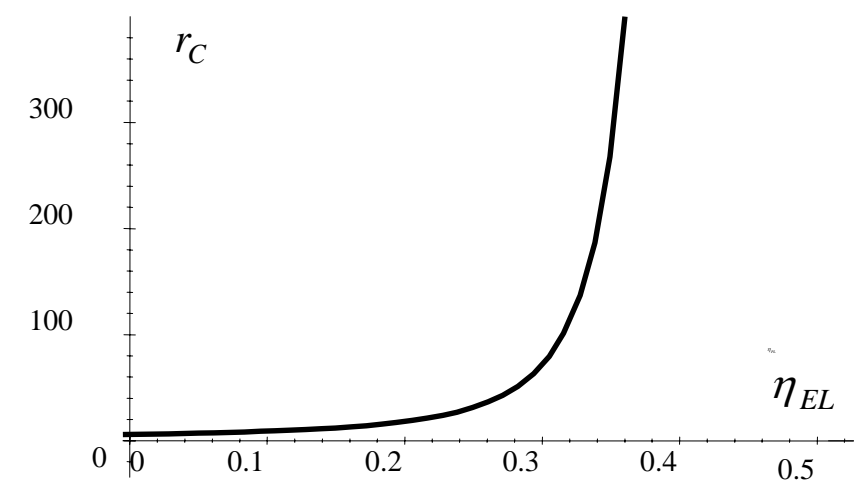

Fig. 3. Variación de $r_{C}$ respecto de $\eta_{E L}$

Por otro lado, dado un valor de $r_{C}$ para los valores de los parámetros utilizados en las figuras 2, 3 y 4 , es posible hallar el comportamiento de $\eta_{I L}$ en función del parámetro $\varepsilon \equiv T_{2} / T_{1}$, donde se observa lo correcto de la afirmación (21), de manera que $\eta_{I L} \rightarrow \eta_{O}$ sólo cuando $\varepsilon \rightarrow 1$, como se observa en la figura 5, para la función ecológica, en donde se ha tomado el valor $r_{C}=10$ cercano a los valores reales de la razón de compresión (ver Burghardt, 1982).

Además, los valores obtenidos con la aproximación lineal se acercan más de lo que se acercan los correspondientes valores $\eta_{C A}$ y $\eta_{E}$, a los valores reportados en las referencias mencionadas. LoS valores físicamente posibles de $r_{C}$ llevan a valores de $\lambda$ que cumplen $0<\lambda<1$. Además, se observa que la razón de compresión es un parámetro importante, para modelar adecuadamente el funcionamiento de una máquina térmica, y es importante tomarlo en cuenta. Desde luego, es difícil introducir un parámetro tal en la modelación de plantas generadoras de electricidad. 


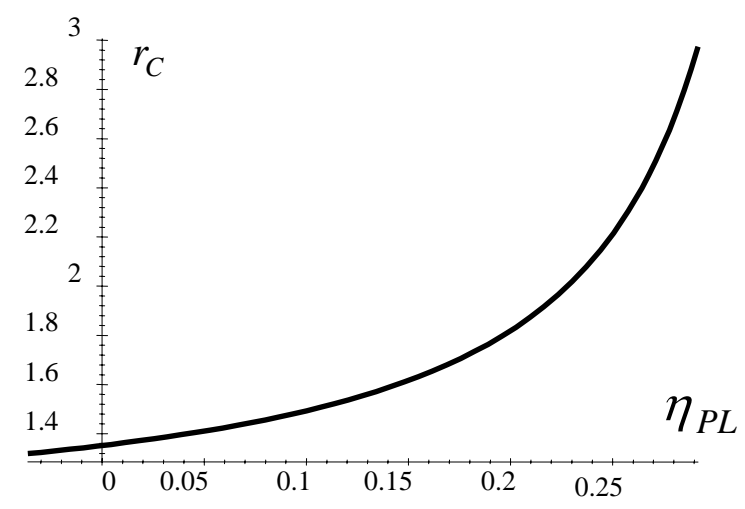

Fig. 4. Sección de la figura 2 encerrada por líneas discontinuas, mostrando el valor mínimo de $r_{C}$ correspondiente a $\eta_{P L}=0$.

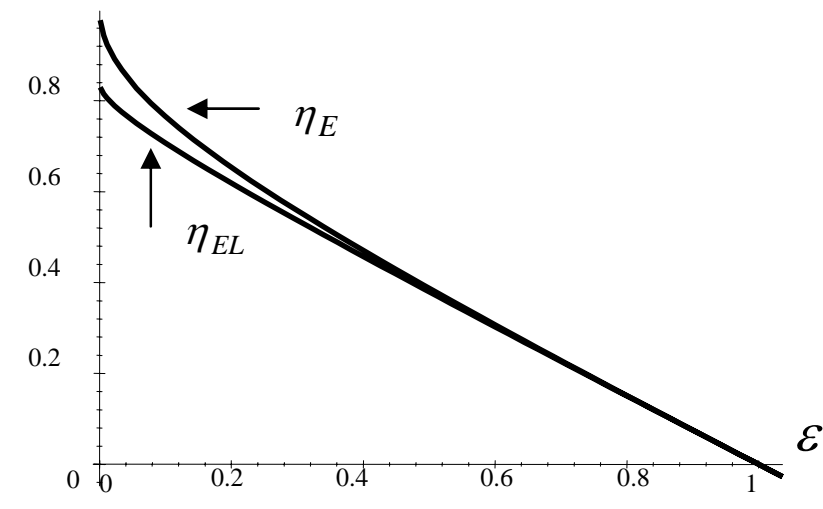

Fig. 5. Comparación entre $\eta_{E}=\eta_{E O}$ y la aproximación lineal $\eta_{E L}$, para el valor $r_{C}=10$

\section{TRANSFERENCIA DE CALOR NO LINEAL}

A partir de la definición de eficiencia $\eta \equiv 1-Q_{C} / Q_{A}$, donde $Q_{C}$ es el calor cedido por el sistema y $Q_{A}$ es el calor absorbido por el sistema, la condición $Q_{1} / T_{1 w}=Q_{2} / T_{2 w}$, condición de endorreversibilidad, permite que la forma de la eficiencia sea en general como $\eta=1-T_{2 W} / T_{1 W}$, aplicable a cualquier ciclo endorreversible. Pero, la razón de temperaturas $T_{2 W} / T_{1 W}$, como una función de la razón de las temperaturas de los almacenes de calor, $T_{2} / T_{1}$, cambia de acuerdo a la función objetivo y a la ley de transferencia de calor asumida. Considérese ahora una ley de transferencia de calor de la forma

$\frac{d Q}{d t}=\alpha\left(T-T_{0}\right)^{k}$

donde $k>1, \alpha$ continúa siendo la conductancia térmica constante, $d Q / d t$ es la razón de calor transferido entre dos subsistemas en contacto térmico, y las temperaturas, $T$ y $T_{0}$, de estos subsistemas cumplen $T>T_{0}$. No es difícil mostrar que utilizando el modelo de Gutkowicz-Krusin et al (1979) y Agrawal y Menon (1990), y una ley de transferencia de calor como en (22), para el mismo ciclo antes considerado, las funciones potencia y función ecológica tienen la forma,

$$
P=T_{1}^{k} \alpha(1-z)(1+\lambda \ln z)\left[\frac{1}{(1-x)^{k}}+\frac{z}{(z x-\varepsilon)^{k}}\right]^{-1}, y, \quad E=T_{1}^{k} \alpha(1+\lambda \ln z)(1+\varepsilon-2 z)\left[\frac{1}{(1-x)^{k}}+\frac{z}{(z x-\varepsilon)^{k}}\right]^{-1} .
$$

Para el caso particular $k=5 / 4$, ley de transferencia de calor de Dulong y Petit, e introduciendo aproximaciones adecuadas para los exponentes de $z$ en las expresiones obtenidas al maximizar las funciones $P$ y $E$, se obtiene expresiones para la eficiencia a orden lineal en el parámetro $\lambda$.

A potencia máxima se encuentra,

$\eta_{L P D P}=1-z_{O P D P}\left[1+\lambda B_{1}(\varepsilon)\right] ; B_{1}(\varepsilon)=\frac{16\left(1-z_{O P D P}\right)\left(\varepsilon-z_{O P D P}\right)}{z_{O P D P}\left(5-4 \varepsilon-40 z_{O P D P}\right)} \quad$ y $z_{O P D P}=\frac{1-\varepsilon+\sqrt{\varepsilon^{2}+98 \varepsilon+1}}{10}$

A función ecológica máxima se encuentra,

$\eta_{L E D P}=1-z_{\text {OEDP }}\left[1+\lambda C_{1}(\varepsilon)\right]$ 
Luna

siendo, $C_{1}(\varepsilon)=\frac{-2 z_{O E D P}+2 \varepsilon-6 z_{O E D P} \varepsilon+2 \varepsilon^{2} 4 z_{O E D P}^{2}}{z_{O E D P}\left(-9 z_{O E D P}-\frac{1}{4} \varepsilon+\frac{1}{4}\right)}, z_{O E D P}=\frac{1-\varepsilon+\sqrt{649 \varepsilon^{2}+646 \varepsilon+1}}{36}$.

La expresión (19) sigue siendo válida, pero ahora debe substituirse la eficiencia obtenida a partir de la utilización de la ley de transferencia de Dulong y Petit. Pueden también compararse las expresiones de las eficiencias mostradas en el presente trabajo, a orden cero, y las expresiones obtenidas por otros autores. Así, para el caso de la aproximación de la eficiencia ecológica, $\eta_{E D P}, y$ la obtenida en Angulo-Brown et al $(1993,1994) \eta_{M E D P}$, se tiene la figura 6.

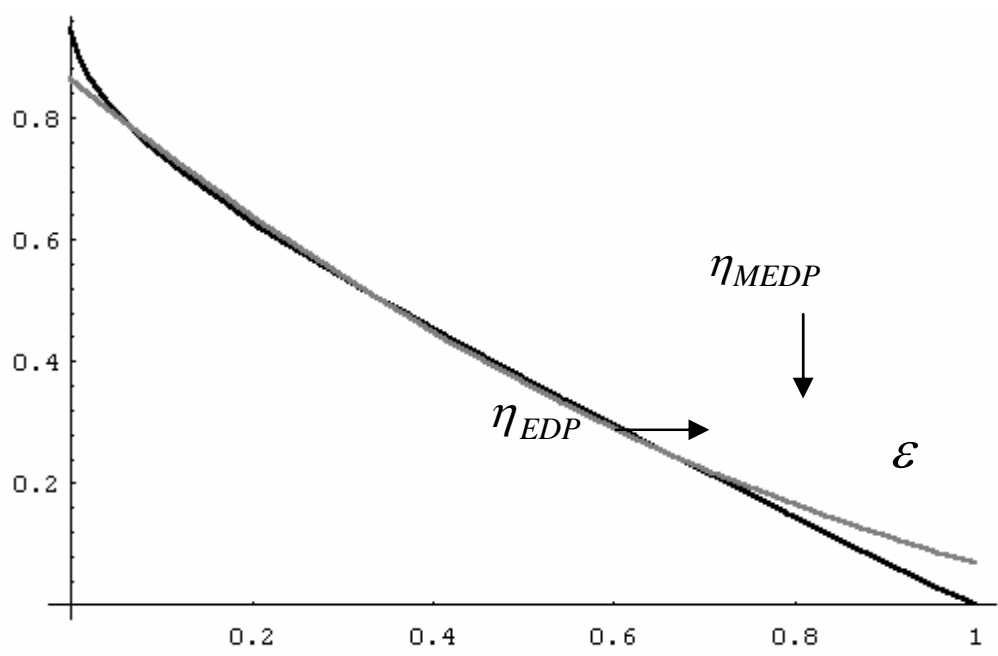

Fig. 6. Comparación de la eficiencia ecológica hallada numéricamente $\eta_{M E D P}$ y la eficiencia ecológica aproximada $\eta_{E D P}$, con ley de transferencia de Dulong y Petit

En los trabajos citados, y en el presente, se ha supuesto un gas ideal como substancia de trabajo. Sin embargo, en la realidad las máquinas térmicas funcionan con gases no ideales, aún más, generalmente son mezclas de gases. En diversos trabajos se ha analizado el ciclo de Curzon y Ahlborn para un gas no ideal como substancia de trabajo; en particular un gas de van der Waals (Ladino-Luna, 2005). La suposición de un gas de van der Waals al mismo tiempo que una ley de transferencia de calor no lineal debe llevar a expresiones más generales que las aquí halladas, para la aproximación lineal de la eficiencia a potencia máxima y a función ecológica máxima.

\section{CONCLUSIONES}

A partir de los resultados obtenidos en Gutkowicz-Krusin et al (1979) y Ladino-Luna y de la Selva (2000), y tomando la aproximación lineal de tales expresiones, el comportamiento de la razón de compresión respecto del parámetro $\varepsilon$ pudo ser analizado, lo que permitió ver que existe una relación entre la razón de compresión y este parámetro. Estos resultados muestran que una adecuada relación entre $r_{C}$ y $\varepsilon$ mejora la eficiencia del modelo utilizado. Una aproximación a orden 2 debe llevar a un mayor acercamiento entre la eficiencia aproximada y los valores reales reportados. La razón de compresión resulta ser un parámetro útil para modelar el funcionamiento de una máquina térmica, pero es difícil introducir un parámetro tal en plantas que generan electricidad. Pudiera hallarse un modelo donde $r_{C}$ pudiese ser incorporada en la forma aquí mostrada.

\section{AGRADECIMIENTOS}

Los autores agradecen el apoyo del CONACYT (México) por el apoyo a través del SNI. 


\section{Luna}

\section{REFERENCIAS}

Agrawal, D.C., J.M. Gordon, y M. Huleihil; Edoreversible Engines with Finite-Time Adiabats. Indian J. Eng. Mat.Sc., 1, 195-198 (1994).

Agrawal, D.C. y V.J. Menon; Finite-Time Carnot Refrigerator with Wail and Products Loads, J. Appl. Phys. 74, 2153-2158 (1993).

Agrawal, D.C. y V.J. Menon; Performance of a Carnot Refrigerator at Maximum Cooling Power. J. Phys. A, 23, 5319-5326 (1990).

Angulo-Brown, F.; An entropy production approach to the Curzon and Ahlborn cycle. Rev. Méx. Fís., 37, 87-96 (1991a).

Angulo-Brown, F.; An Ecological Optimization Criterion for Finite-time Heat Engines. J. Appl. Phys., 69, 7465-7469 (1991b).

Angulo-Brown, F. y R.T. Páez-Hernández; Endoreversible Thermal Cycles with a Nonlinear Heat Transfer Law, J. Appl. Phys., 74, 2216-2219 (1993)

Arias-Hernández, L.A. y F. Angulo-Brown; Thermodynamic Optimization of Endoreversible Engines, Rev. Mex. Fís., 40, 866-877 (1994).

Burghardt, M.D.; Engineering Thermodynamics, Harper and Row, N. Y. (1982) section 5.2

Curzon, F. L. y B. Ahlborn; Efficiency of a Carnot engine at maximum power output, Am. J. Phys., 43, 22-24 (1975).

Chen, L.F. Sun y Ch. Wu; Effect of Heat Transfer Law on the Performance of a Generalized Irreversible Carnot Engine, J. Phys. D: Appl. Phys., 32, 99-105 (1999).

Gutkowicz-Krusin, D., I. Procaccia, y J. Ross; On the Efficiency of rate processes. Power and efficiency of heat engines, J. Chem. Phys. 69, 3898-3906 (1978).

Ladino-Luna, D; Van der Waals Gas as Working Substance in a Curzon and Ahlborn-Novikov Engine, Entropy, 7, 108-121 (2005).

Ladino-Luna, D; Approximate Ecological Efficiency with a Non-Linear Heat Transfer, J. of the Energy Institute, 81, 114-117 (2008).

Ladino-Luna, D. y S.M.T. de la Selva; The Ecological Efficiency of a Thermal Finite Time Engine, Rev. Mex. Fís., 46, 52-56 (2000).

Ondrechen, M.O., M.H. Rubin y Y. B. Band; The Generalized Carnot Cycle; a Working Fluid Operating in Finite Time Between Finite Time Heat Sorces and Sinks, J. Chem. Phys., 78, 4721-4727 (1983).

Rubin, M.; Optimal Configuration of a Class of Irreversible Heat Engines. I, Phys. Rev. A, 19, 12721276 (1979).

Rubin, M.; Optimal Configuration of a Class of Irreversible Heat Engines. II. Phys. Rev. A. 19, $1277-$ 1288 (1979).

Rubin, M; Optimal Configuration of an Irreversible Heat Engine with Fixed Compression Ratio. Phys. Rev. A, 22, 1741-1752 (1980). 
Salamon, P., B. Andresen, y R.S. Berry, Thermodynamics in Finite Time. I. The Step-Carnot Cycle, Phys. Rev. A, 15, 2086-2093 (1977).

Salamon, P., B. Andresen, y R.S. Berry; Thermodynamics in Finite Time. II. Potentials for Finite-time processes Phys. Rev. A, 15, 2094-2102 (1977).

Salamon, P., A. Nitzan, B. Andresen, y R.S. Berry; Minimum Entropy Production and the Optimization of Heat Engines. Phys. Rev. A. 21, 2115-2129 (1980).

Torres, J.L.; Minimal Rate of Entropy Production as a Criterion of Merit for Thermal Engines. Rev. Mex. Fís., 34, 18-24 (1988).

Yan, Z. y J. Chen; A Class of Irreversible Carnot Refrigeration Cycles with a General Heat Transfer Law, J. Phys D: Appl. Phys., 23, 136-141 (1990). 